\title{
ITERATIVE REWEIGHTED ALGORITHMS FOR JOINT USER IDENTIFICATION AND CHANNEL ESTIMATION IN SPATIALLY CORRELATED MASSIVE MTC
}

\author{
Hamza Djelouat, Markus Leinonen, and Markku Juntti \\ Centre for Wireless Communications - Radio Technologies, FI-90014, University of Oulu, Finland
}

\begin{abstract}
Joint user identification and channel estimation (JUICE) is a main challenge in grant-free massive machine-type communications (mMTC). The sparse pattern in users' activity allows to solve the JUICE as a compressed sensing problem in a multiple measurement vector (MMV) setup. This paper addresses the JUICE under the practical spatially correlated fading channel. We formulate the JUICE as an iterative reweighted $\ell_{2,1}$-norm optimization. We develop a computationally efficient alternating direction method of multipliers (ADMM) approach to solve it. In particular, by leveraging the second-order statistics of the channels, we reformulate the JUICE problem to exploit the covariance information and we derive its ADMM-based solution. The simulation results highlight the significant improvements brought by the proposed approach in terms of channel estimation and activity detection performances.
\end{abstract}

Index Terms - mMTC, ADMM, user identification, channel estimation, spatially correlated channels.

\section{INTRODUCTION}

The demand for internet of things (IoT) applications drives the deployment of massive machine-type communications (mMTC) as a major use case in 5G wireless technologies. mMTC implies sporadic uplink communication from a massive number of IoT devices, called user equipments (UEs). Therefore, communications with low signalling overhead is needed. Therefore, grant-free access has been identified as a key enabler for mMTC [1]. It requires joint identification of the active UEs and estimation of their channel state information (CSI), known as the joint user identification and channel estimation (JUICE) problem.

The sparse user activity pattern along with the multi-antenna base station (BS) setup motivates the formulation of JUICE as a compressed sensing (CS) [2] problem in a multiple measurement vector (MMV) setup. The optimal solution for sparse signal recovery requires solving an NP-hard $\ell_{0}$-norm minimization problem. Therefore, several approaches have been proposed to overcome this limitation, e.g., mixed norm minimization [3] (and the references therein), iterative algorithms [4 5], and sparse Bayesian learning (SBL) [6].

In the context of grant-free based JUICE, the existing works focus on techniques based on greedy algorithms [7. 8], approximate message passing [9]-12], SBL [13], and maximum likelihood estimation [14]. Nevertheless, since the aforementioned works assume that the channel components are independent, the performance of JUICE may deteriorate as this assumption is not always practical [15].

This work has been financially supported in part by the Academy of Finland (ROHM project, grant 319485) and Academy of Finland 6Genesis Flagship (grant 318927). The work of M. Leinonen has also been financially supported in part by Infotech Oulu and the Academy of Finland (grant 323698).
In this paper, we formulate the JUICE as an iterative reweighted $\ell_{2,1}$-norm minimization. While the $\ell_{1}$-norm penalty follows from the conventional approximation of $\ell_{0}$-norm to relax the JUICE into a tractable convex problem, the reweighting compensates for the key difference between the $\ell_{1}$ and $\ell_{0}$-norms: the dependency on coefficients' amplitude [16]. Moreover, differently from the assumption of uncorrelated channels as in the aforementioned works [7.-11 13 14], this paper addresses the JUICE in the more practical spatially correlated multiple-input multiple-output (MIMO) channels. In such a channel model, the channel spatial correlation varies slowly compared to the channel realizations, hence, they can be estimated with high accuracy in practice [17]. Thus, the spatial correlation information can be exploited to enhance the JUICE performance.

The main contributions of this paper are summarized as follows. First, when the second-order statistics of the channels are not available, we formulate the JUICE as an iterative reweighted $\ell_{2,1^{-}}$ norm minimization and we derive a computationally efficient solution based on alternating direction method of multipliers (ADMM) by providing a closed-form expression to each sub-problem at each iteration. Second, when the BS knows the channels' second-order statistics, we augment the optimization problem with a penalty term on the deviation of the covariance matrices of the estimated channels from their respective true covariance matrices. Furthermore, once the active UEs are identified, a minimum mean square error (MMSE) estimator is deployed to improve channel estimation. The proposed approaches are empirically shown to significantly improve the JUICE performance.

\section{SYSTEM MODEL}

Consider a single cell uplink communication scheme with a single BS that is equipped with a uniform linear array (ULA) containing $M$ antennas surrounded by a set of $N$ single-antenna UEs $\mathcal{N}=\{1, \ldots, N\}$. We consider a block fading channel over each coherence period $T_{\mathrm{c}}$. The channel response $\mathbf{h}_{i} \in \mathbb{C}^{M}$ between the $i$ th UE and the BS is modelled as

$$
\mathbf{h}_{i}=\frac{1}{\sqrt{P_{i}}} \sum_{p=1}^{P_{i}} \omega_{i, p} \mathbf{a}\left(\psi_{i, p}\right), \quad \forall i \in \mathcal{N},
$$

where $P_{i}$ is the number of physical signal paths, $\omega_{i, p} \in \mathbb{C}$ accounts for the $p$ th path gain and $\mathbf{a}\left(\psi_{i, p}\right) \in \mathbb{C}^{M}$ is the array response of the ULA given as $\left[\mathbf{a}\left(\psi_{i, p}\right)\right]_{m}=e^{-j(m-1) 2 \pi \Delta_{\mathrm{r}} \cos \left(\psi_{i, p}\right)}$, $m=1, \ldots, M$, where $\Delta_{\mathrm{r}}$ is the normalized space between each pair of BS antennas, and $\psi_{i, p}$ is the angle of arrival of the $l$ th path [18].

At each coherence interval $T_{\mathrm{c}}$, a new and independent channel realization $\mathbf{h}_{i}$ is observed in (1). The channels are considered to be wide-sense stationary [17], i.e., the channel covariance matrix of the $i$ th UE, denoted as $\mathbf{R}_{i}=\mathbb{E}\left[\mathbf{h}_{i} \mathbf{h}_{i}^{\mathrm{H}}\right] \in \mathbb{C}^{M \times M}$, varies in a slower time-scale compared to the channel realizations and it remains fixed for $\tau_{\mathrm{s}}$ coherence intervals, where $\tau_{\mathrm{s}}$ can be on the order of thousands 
[15 19]. We assume the common convention that the covariance matrices $\left\{\mathbf{R}_{i}\right\}_{i=1}^{N}$ are known by the BS [17].

Due to the sporadic nature of mMTC, only $K \ll N$ UEs are active at each $T_{\mathrm{c}}$. Therefore, for coherent data detection, the active UEs have to be detected and their channels have to be estimated. To this end, the BS assigns to each UE $i \in \mathcal{N}$ a unit-norm pilot sequence $\phi_{i} \in \mathbb{C}^{\tau_{\mathrm{p}}}$. To mitigate the channel gain difference between the UEs, a power control policy is deployed such that UE $i$ transmits with a power $\rho_{i}$ that is inversely proportional to the average channel gain 15]. We define the pilot matrix as $\boldsymbol{\Phi}=\left[\boldsymbol{\phi}_{1}, \ldots, \boldsymbol{\phi}_{N}\right] \in \mathbb{C}^{\tau_{\mathrm{p}} \times N}$ and the effective channel matrix as $\mathbf{X}=\left[\mathbf{x}_{1}, \ldots, \mathbf{x}_{N}\right]^{\mathrm{T}} \in \mathbb{C}^{N \times M}$, where $\mathbf{x}_{i}=\gamma_{i} \sqrt{\rho_{i}} \mathbf{h}_{i}$ is the effective channel for $i$ th UE and $\gamma_{i}$ is an activity indicator, defined as

$$
\gamma_{i}= \begin{cases}1, & i \in \mathcal{S} \\ 0, & \text { otherwise }\end{cases}
$$

where $\mathcal{S} \subseteq \mathcal{N},|\mathcal{S}|=K$, denotes the set of active UEs.

During each $T_{\mathrm{c}}$, the $K$ active UEs transmit their pilot sequences to the $\mathrm{BS}$, and the received pilot signal $\mathbf{Y} \in \mathbb{C}^{\tau_{\mathrm{p}} \times M}$ is given by

$$
\mathbf{Y}=\mathbf{\Phi} \mathbf{X}+\mathbf{W}
$$

where $\mathbf{W} \in \mathbb{C}^{\tau_{\mathrm{p}} \times M}$ is additive white Gaussian noise with independent and identically distributed (i.i.d.) elements as $\mathcal{C N}\left(0, \sigma^{2}\right)$,

\section{PROPOSED SOLUTION VIA ITERATIVE APPROACH}

\subsection{JUICE via Reweighted $\ell_{2,1}$-Norm Minimization}

Since the rows of the effective channel matrix $\mathbf{X}$ in (2) corresponding to the inactive UEs are zero, $\mathbf{X}$ has a row-sparse structure. Thus, JUICE can be modeled as a joint sparse MMV reconstruction problem. The optimal sparse recovery requires solving a combinatorial NP-hard $\ell_{0}$-norm minimization problem. Thus, a convex relaxation in the form of $\ell_{2,1}$-norm is considered in practice to obtain a computationally tractable problem, commonly formulated as

$$
\min _{\mathbf{X}} \frac{1}{2}\|\mathbf{\Phi} \mathbf{X}-\mathbf{Y}\|_{\mathrm{F}}^{2}+\beta_{1}\|\mathbf{X}\|_{2,1} \text {. }
$$

However, unlike the democratic $\ell_{0}$-norm where the non-zero coefficients are penalized equally, $\ell_{1}$-norm is biased toward larger magnitudes, i.e., coefficients with large amplitude are penalized more heavily than smaller ones [16]. Therefore, striving for a better recovery, we use the $\log$-sum penalty to relax the $\ell_{0}$-norm as

$$
\begin{array}{ll}
\min _{\mathbf{X}, \mathbf{u}} & \frac{1}{2}\|\mathbf{\Phi} \mathbf{X}-\mathbf{Y}\|_{\mathrm{F}}^{2}+\beta_{1} \sum_{i=1}^{N} \log \left(u_{i}+\epsilon_{0}\right) \\
\text { s.t. } & \left\|\mathbf{x}_{i}\right\|_{2} \leq u_{i}, \forall i \in \mathcal{N} .
\end{array}
$$

The log-sum penalty resembles most closely the $\ell_{0}$-norm penalty when $\epsilon_{0} \rightarrow 0$. However, a practical choice is to set $\epsilon_{0}$ to be slightly less than the expected amplitude of the non-zero rows in $\mathbf{X ~ [ 1 6 ] . ~}$

The optimization problem in (4) is a sum of a convex and a concave function, thus, it is not convex in general. Therefore, we rely on majorization-minimization (MM) approach and we approximate the concave penalty by its first-order Taylor expansion. Subsequently, we solve (4) as an iterative reweighted problem given as

$$
\mathbf{X}^{(l+1)}=\min _{\mathbf{X}} \sum_{i=1}^{N} \beta_{1} g_{i}^{(l)}\left\|\mathbf{x}_{i}\right\|_{2}+\frac{1}{2}\|\mathbf{\Phi} \mathbf{X}-\mathbf{Y}\|_{\mathrm{F}}^{2},
$$

where $(l)$ denotes the MM iteration and $g_{i}^{(l)}=\left(\epsilon_{0}+\left\|\mathbf{x}_{i}^{(l)}\right\|_{2}\right)^{-1}$.

The problem in (5) is convex and can be solved optimally utilizing standard convex optimization techniques. However, as the mMTC system may grow large, the standard techniques may not be computationally efficient. Thus, we propose the use of ADMM to solve the optimization problem in (5) at each MM iteration $(l)$.

Specifically, we introduce an auxiliary variable $\mathbf{Z} \in \mathbb{C}^{M \times N}$ and the dual variable $\boldsymbol{\Lambda} \in \mathbb{C}^{M \times N}$, hence, the augmented Lagrangian of (5) is given by

$$
\min _{\mathbf{X}, \mathbf{Z}} \beta_{1} \sum_{i=1}^{N} g_{i}^{(l)}\left\|\mathbf{x}_{i}\right\|_{2}+\frac{1}{2}\|\mathbf{\Phi} \mathbf{Z}-\mathbf{Y}\|_{\mathrm{F}}^{2}+\frac{\rho}{2}\left\|\mathbf{X}-\mathbf{Z}+\frac{\boldsymbol{\Lambda}}{\rho}\right\|_{\mathrm{F}}^{2}-\frac{\|\boldsymbol{\Lambda}\|_{\mathrm{F}}^{2}}{2 \rho},
$$

where $\rho$ is a positive parameter. The ADMM solves the optimization problem through sequential updates of $(\mathbf{X}, \mathbf{Z}, \mathbf{\Lambda})$ as follows [20]:

$$
\begin{aligned}
\mathbf{Z}^{(k+1)}:=\min _{\mathbf{Z}} \frac{1}{2}\|\mathbf{\Phi} \mathbf{Z}-\mathbf{Y}\|_{\mathrm{F}}^{2}+\frac{\rho}{2}\left\|\mathbf{X}^{(k)}-\mathbf{Z}+\frac{1}{\rho} \boldsymbol{\Lambda}^{(k)}\right\|_{\mathrm{F}}^{2} \\
\mathbf{X}^{(k+1)}:=\min _{\mathbf{X}} \sum_{i=1}^{N} \beta_{1} g_{i}^{(l)}\left\|\mathbf{x}_{i}\right\|_{2}+\frac{\rho}{2}\left\|\mathbf{X}-\mathbf{Z}^{(k+1)}+\frac{1}{\rho} \boldsymbol{\Lambda}^{(k)}\right\|_{\mathrm{F}}^{2} \\
\boldsymbol{\Lambda}^{(k+1)}:=\boldsymbol{\Lambda}^{(k)}+\rho\left(\mathbf{X}^{(k+1)}-\mathbf{Z}^{(k+1)}\right) .
\end{aligned}
$$

where the superscript $(k)$ denotes the ADMM iteration index. The derivations of the ADMM steps (7) and (8) are detailed below.

The Z Z-update step in (7) solves a convex optimization problem. Thus, $\mathbf{Z}^{(k+1)}$ is obtained by setting the gradient of the objective function in (7) with respect to $\mathbf{Z}$ to zero, resulting in

$$
\mathbf{Z}^{(k+1)}=\left(\rho \mathbf{X}^{(k)}+\boldsymbol{\Lambda}^{(k)}+\mathbf{Y}^{\mathrm{T}} \boldsymbol{\Phi}^{*}\right)\left(\boldsymbol{\Phi}^{\mathrm{T}} \boldsymbol{\Phi}^{*}+\rho \mathbf{I}_{N}\right)^{-1},
$$

where $(\cdot)^{*}$ denotes the complex conjugate operator. Note that the inversion $\left(\boldsymbol{\Phi}^{*} \boldsymbol{\Phi}^{\mathrm{T}}+\rho \mathbf{I}_{N}\right)^{-1}$ can be computed once and stored to expedite the $\mathbf{Z}$-update step.

Next, the $\mathrm{X}$-update in 8 can be decomposed into $N$ subproblems as follows

$$
\mathbf{x}_{i}^{(k+1)}:=\min _{\mathbf{x}_{i}} \frac{\beta_{1} g_{i}^{(l)}}{\rho}\left\|\mathbf{x}_{i}\right\|_{2}+\frac{1}{2}\left\|\mathbf{x}_{i}-\mathbf{c}_{i}^{(k)}\right\|_{2}^{2}, \quad \forall i \in \mathcal{N},
$$

where $\mathbf{c}_{i}^{(k)}=\mathbf{z}_{i}^{(k+1)}-\frac{1}{\rho} \boldsymbol{\lambda}_{i}^{(k)}$ and $\boldsymbol{\lambda}_{i}^{(k)}$ is the $i$ th column of $\boldsymbol{\Lambda}^{(k)}$. The problem in (11) admits the closed-form solution given by [21]

$$
\mathbf{x}_{i}^{(k+1)}=\frac{\max \left\{0,\left\|\mathbf{c}_{i}^{(k)}\right\|_{2}-\frac{\beta_{1} g_{i}^{(l)}}{\rho}\right\}}{\left\|\mathbf{c}_{i}^{(k)}\right\|_{2}} \mathbf{c}_{i}^{(k)}, \quad \forall i \in \mathcal{N} .
$$

\subsection{Covariance Aided JUICE}

Although the sparsity of the matrix $\mathbf{X}$ is utilized in (4), the information embedded in channel covariance matrices available at the BS is neglected. On this account, we reformulate the problem in (4) so that it exploits also the covariance information. The key idea is that the sample covariance matrix $\mathbf{x}_{i} \mathbf{x}_{i}^{\mathrm{H}}$ for each active UE $i \in \mathcal{S}$ carries similar information as the true scaled covariance matrix $\tilde{\mathbf{R}}_{i}=\rho_{i} \mathbf{R}_{i}$.

Based on the above arguments, we augment the optimization problem in (4) with a regularization term that penalizes the deviation of the sample covariance matrix $\mathbf{x}_{i} \mathbf{x}_{i}^{\mathrm{H}}$ from the true scaled covariance matrix $\tilde{\mathbf{R}}_{i}$. Thus, the covariance aided JUICE problem is expressed as follows

$$
\begin{aligned}
& \min _{\mathbf{X}, \mathbf{u}} \frac{1}{2}\|\mathbf{\Phi} \mathbf{X}-\mathbf{Y}\|_{\mathrm{F}}^{2}+\beta_{1} \sum_{i=1}^{N} \log \left(u_{i}+\epsilon_{0}\right) \\
& +\beta_{2} \sum_{i=1}^{N} \mathbb{I}\left(u_{i}\right)\left\|\mathbf{x}_{i} \mathbf{x}_{i}^{\mathrm{H}}-\tilde{\mathbf{R}}_{i}\right\|_{\mathrm{F}}^{2} \quad \text { s.t. }\left\|\mathbf{x}_{i}\right\|_{2} \leq u_{i}, \forall i \in \mathcal{N},
\end{aligned}
$$

where $\beta_{2}$ controls the penalty on the covariance deviation term and $\mathbb{I}(\cdot)$ is an indicator function given by

$$
\mathbb{I}\left(u_{i}\right)= \begin{cases}1, & u_{i}>0 \\ 0, & u_{i}=0\end{cases}
$$


Note that $\mathbb{I}\left(u_{i}\right)$ ensures that only the estimated active UEs are penalized with the covariance regularization term. The indicator function is hard to handle due to its combinatorial nature. Therefore, we relax 14 with a function $f(\cdot)$ that approximates the sign step functions for positive values $v$, i.e., we define

$$
f(v ; \kappa)=\frac{\log (1+\kappa v)}{\log (1+\kappa)},
$$

where $\kappa$ is a positive parameter to adjust the steepness of the function for small input values [22]. Subsequently, 13 is relaxed as

$$
\begin{aligned}
& \min _{\mathbf{X}, \mathbf{u}} \frac{1}{2}\|\mathbf{\Phi} \mathbf{X}-\mathbf{Y}\|_{\mathrm{F}}^{2}+\beta_{1} \sum_{i=1}^{N} \log \left(u_{i}+\epsilon_{0}\right) \\
& +\beta_{2} \sum_{i=1}^{N} f\left(u_{i} ; \kappa\right)\left\|\mathbf{x}_{i} \mathbf{x}_{i}^{\mathrm{H}}-\tilde{\mathbf{R}}_{i}\right\|_{\mathrm{F}}^{2} \quad \text { s.t. } \quad\left\|\mathbf{x}_{i}\right\|_{2} \leq u_{i}, \forall i \in \mathcal{N} .
\end{aligned}
$$

Since both the log-sum penalty and $f\left(u_{i} ; \kappa\right)$ are concave functions, we rely on the MM approach and we approximate the problem in (16) by its first-order Taylor expansion at $\mathbf{u}^{(l)}$. Subsequently, with the use of some simple manipulations, we can solve (16) as the following iterative reweighted problem given at $l$ th MM iteration by

$$
\begin{aligned}
\mathbf{X}^{(l+1)}= & \min _{\mathbf{X}} \sum_{i=1}^{N} \beta_{1} g_{i}^{(l)}\left\|\mathbf{x}_{i}\right\|_{2}+\frac{1}{2}\|\mathbf{\Phi} \mathbf{X}-\mathbf{Y}\|_{\mathrm{F}}^{2} \\
& +\beta_{2} \sum_{i=1}^{N} q_{i}^{(l)}\left\|\mathbf{x}_{i}\right\|_{2}\left\|\mathbf{x}_{i} \mathbf{x}_{i}^{\mathrm{H}}-\tilde{\mathbf{R}}_{i}\right\|_{\mathrm{F}}^{2}
\end{aligned}
$$

with $q_{i}^{(l)}=\frac{\kappa}{\log (1+\kappa)} \frac{1}{1+\kappa\left\|\mathbf{x}_{i}^{(l)}\right\|_{2}}, \forall i \in \mathcal{N}$.

The objective function in $(17)$ is non-convex due to the covariance deviation penalty term. Therefore, in order to overcome the non-convexity, we introduce the splitting variables $\mathbf{Z}$ and $\mathbf{V}$ and we rewrite the objective function in (17) as

$$
\begin{aligned}
& \min _{\mathbf{X}} \sum_{i=1}^{N} \beta_{1} g_{i}^{(l)}\left\|\mathbf{x}_{i}\right\|_{2}+\frac{1}{2}\|\mathbf{\Phi} \mathbf{Z}-\mathbf{Y}\|_{\mathrm{F}}^{2}+ \\
& \beta_{2} \sum_{i=1}^{N} q_{i}^{(l)}\left\|\mathbf{x}_{i}\right\|_{2}\left\|\mathbf{z}_{i} \mathbf{v}_{i}^{\mathrm{H}}-\tilde{\mathbf{R}}_{i}\right\|_{\mathrm{F}}^{2} \\
& \text { s.t. } \quad \mathbf{x}_{i}=\mathbf{z}_{i}, \quad \mathbf{x}_{i}=\mathbf{v}_{i}, \forall i \in \mathcal{S}
\end{aligned}
$$

The optimization problem in (18) is block multi-convex, thus, we utilize ADMM to solve it efficiently. Accordingly, the augmented Lagrangian associated with (18) is given by

$$
\begin{aligned}
& \min _{\mathbf{Z}, \mathbf{V}, \mathbf{X}} \frac{1}{2}\|\mathbf{\Phi} \mathbf{Z}-\mathbf{Y}\|_{\mathrm{F}}^{2}+\beta_{2} \sum_{i=1}^{N} q_{i}^{(l)}\left\|\mathbf{x}_{i}\right\|_{2}\left\|\mathbf{z}_{i} \mathbf{v}_{i}^{\mathrm{H}}-\tilde{\mathbf{R}}_{i}\right\|_{\mathrm{F}}^{2} \\
& +\sum_{i=1}^{N} \beta_{1} g_{i}^{(l)}\left\|\mathbf{x}_{i}\right\|_{2}+\frac{\rho}{2}\left\|\mathbf{X}-\mathbf{Z}+\frac{\boldsymbol{\Lambda}_{\mathbf{z}}}{\rho}\right\|_{\mathrm{F}}^{2}+\frac{\rho}{2}\left\|\mathbf{X}-\mathbf{V}+\frac{\boldsymbol{\Lambda}_{\mathrm{V}}}{\rho}\right\|_{\mathrm{F}}^{2} \\
& -\frac{\left\|\boldsymbol{\Lambda}_{\mathrm{V}}\right\|_{\mathrm{F}}^{2}}{2 \rho}-\frac{\left\|\boldsymbol{\Lambda}_{\mathbf{z}}\right\|_{\mathrm{F}}^{2}}{2 \rho}
\end{aligned}
$$

where $\boldsymbol{\Lambda}_{\mathrm{z}}=\left[\boldsymbol{\lambda}_{\mathrm{z}_{1}}, \ldots, \boldsymbol{\lambda}_{\mathrm{z}_{N}}\right]$ and $\boldsymbol{\Lambda}_{\mathrm{v}}=\left[\boldsymbol{\lambda}_{\mathrm{v}_{1}}, \ldots, \boldsymbol{\lambda}_{\mathrm{v}_{N}}\right]$ are the ADMM dual variables. Similarly to (7)- (9), ADMM updates sequentially the primal variables $\mathbf{Z}, \mathbf{V}$ and $\mathbf{X}$ then the dual variables $\boldsymbol{\Lambda}_{\mathrm{z}}$ and $\boldsymbol{\Lambda}_{\mathrm{v}}$.

First, the $\mathbf{Z}$-subproblem, i.e., minimizing 19 with respect to $\mathbf{Z}$, is given by

$$
\begin{aligned}
\mathbf{Z}^{(k+1)}:= & \left.\min _{\mathbf{Z}} \frac{1}{2}\|\mathbf{\Phi} \mathbf{Z}-\mathbf{Y}\|_{\mathrm{F}}^{2}+\beta_{2} q_{i}^{(l)} \sum_{i=1}^{N}\left\|\mathbf{x}_{i}^{(k)}\right\|_{2} \| \mathbf{z}_{i} \mathbf{v}_{i}^{(k)}\right)^{\mathrm{H}}-\tilde{\mathbf{R}}_{i} \|_{\mathrm{F}}^{2} \\
& +\frac{\rho}{2}\left\|\mathbf{X}^{(k)}-\mathbf{Z}+\frac{\boldsymbol{\Lambda}_{\mathbf{z}}^{(k)}}{\rho}\right\|_{\mathrm{F}}^{2} .
\end{aligned}
$$

The objective function in 20] is convex and the solution is obtained by setting the gradient with respect to $\mathbf{Z}$ to zero, resulting in

$$
\mathbf{Z}^{(k+1)}=\left(\mathbf{Y}^{\mathrm{T}} \boldsymbol{\Phi}^{*}+\mathbf{B}^{(k)}\right)\left(\boldsymbol{\Phi}^{\mathrm{T}} \boldsymbol{\Phi}^{*}+\mathbf{D}^{(k)}\right)^{-1} .
$$

where $\mathbf{b}_{i}^{(k)}=2 \beta_{2} q_{i}^{(l)}\left\|\mathbf{x}_{i}^{(k)}\right\|_{2} \tilde{\mathbf{R}}_{i} \mathbf{v}_{i}^{(k)}+\rho \mathbf{x}_{i}^{(k)}+\boldsymbol{\lambda}_{\mathbf{z}_{i}}^{(k)}$ is the $i$ th column of matrix $\mathbf{B}^{(k)}$ and the matrix $\mathbf{D}^{(k)}$ is a diagonal matrix with entries $d_{i}^{(k)}=2 \beta_{2} q_{i}^{(l)}\left\|\mathbf{x}_{i}^{(k)}\right\|_{2}\left\|\mathbf{v}_{i}^{(k)}\right\|_{2}^{2}+\rho$.

Second, the $\mathbf{V}$-update solves the minimization problem given by

$\min _{\mathbf{V}} \beta_{2} \sum_{i=1}^{N} q_{i}^{(l)}\left\|\mathbf{x}_{i}^{(k)}\right\|_{2}\left\|\mathbf{z}_{i}^{(k+1)} \mathbf{v}_{i}^{\mathrm{H}}-\tilde{\mathbf{R}}_{i}\right\|_{\mathrm{F}}^{2}+\frac{\rho}{2}\left\|\mathbf{X}^{(k)}-\mathbf{V}+\frac{\boldsymbol{\Lambda}_{\mathrm{v}}^{(k)}}{\rho}\right\|_{\mathrm{F}}^{2}$.

The optimization problem in 22) can be decoupled into $N$ convex sub-problems, with a unique solution given by:

$$
\mathbf{v}_{i}^{(k+1)}=\frac{2 \beta_{2} q_{i}^{(l)}\left\|\mathbf{x}_{i}^{(k)}\right\|{ }_{2} \tilde{\mathbf{R}}_{i} \mathbf{z}_{i}^{(k+1)}+\rho \mathbf{x}_{i}^{(k)}+\boldsymbol{\lambda}_{\mathbf{v}_{i}}^{(k)}}{2 \beta_{2} q_{i}^{(l)}\left\|\mathbf{x}_{i}^{(k)}\right\|_{2}\left\|\mathbf{z}_{i}^{(k+1)}\right\|_{2}^{2}+\rho}, \forall i \in \mathcal{N} .
$$

Next, with some manipulations, the $\mathbf{X}$-update solves the following convex optimization problem

$$
\mathbf{X}^{(k+1)}:=\min _{\mathbf{X}} \sum_{i=1}^{N} \alpha_{i}^{(k)}\left\|\mathbf{x}_{i}\right\|_{2}+\rho\left\|\mathbf{X}-\mathbf{S}^{(k)}\right\|_{\mathrm{F}}^{2},
$$

where $\mathbf{S}^{(k)}=\frac{1}{2}\left(\mathbf{Z}^{(k+1)}+\mathbf{V}^{(k+1)}-\frac{\boldsymbol{\Lambda}_{\mathrm{z}}^{(k)}+\boldsymbol{\Lambda}_{\mathrm{v}}^{(k)}}{\rho}\right)$ and $\alpha_{i}^{(k)}=$ $\beta_{1} g_{i}^{(l)}+\beta_{2} q_{i}^{(l)}\left\|\mathbf{z}_{i}^{(k+1)} \mathbf{v}_{i}^{(k+1)^{\mathrm{H}}}-\tilde{\mathbf{R}}_{i}\right\|_{\mathrm{F}}^{2}$. The optimal solution to 24] has a closed-form expression given by

$$
\mathbf{x}_{i}^{(k+1)}=\frac{\max \left\{0,\left\|\mathbf{s}_{i}^{(k)}\right\|_{2}-\frac{\alpha_{i}^{(k)}}{2 \rho}\right\}}{\left\|\mathbf{s}_{i}^{(k)}\right\|_{2}} \mathbf{s}_{i}^{(k)}, \quad \forall i \in \mathcal{N} .
$$

The details of the proposed covariance aided JUICE, termed as cov-ADMM, are summarized in Algorithm 1. Note that if the second-order channel statistics are not available, we set $\beta_{2}=0$, hence, Algorithm 1 presents the proposed iterative reweighted ADMM (IRW-ADMM) in Section 3.1 Moreover, if $\beta_{2}=0$ and $q_{i}^{(l)}=g_{i}^{(l)}=1$, for $i \in \mathcal{N}, l=1,2, \ldots$, Algorithm 1 presents the ADMM solution, which we call ADMM, for the problem in (3).

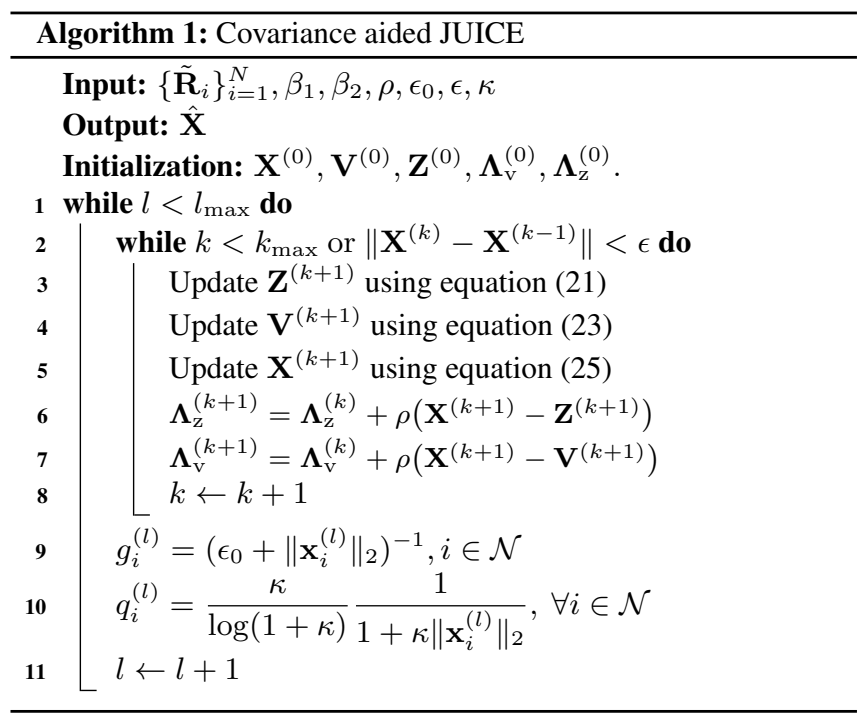




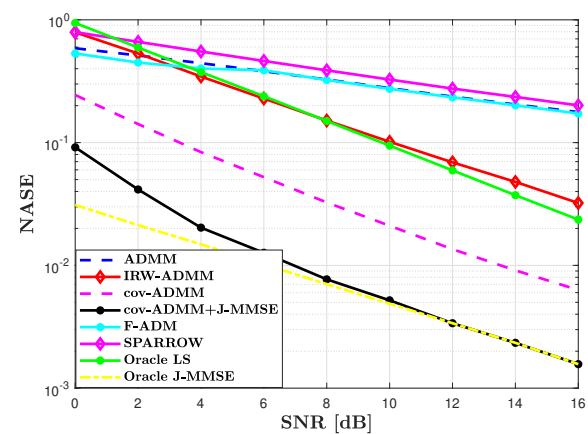

(a) NASE versus SNR.

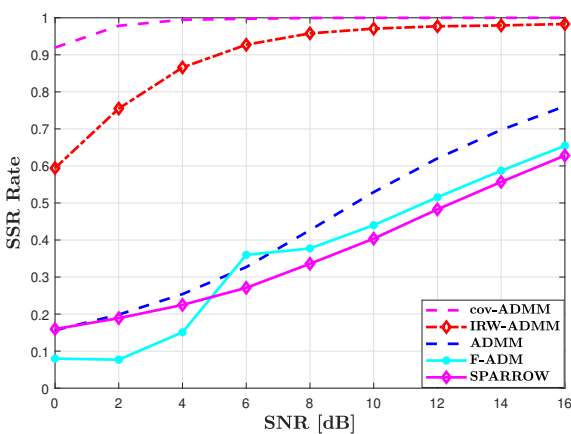

(b) SSR rate versus SNR.

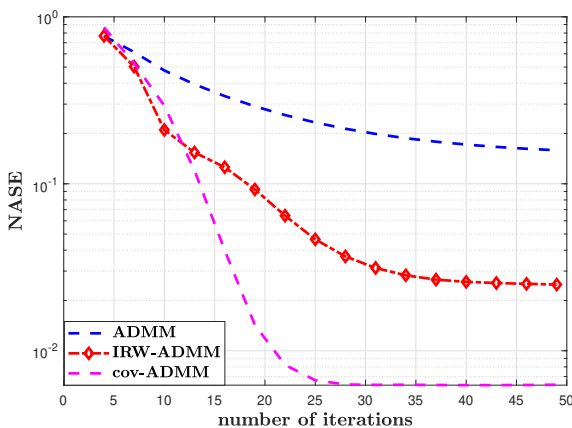

(c) NASE versus the number of iterations.

Fig. 1: Performance comparison for the different algorithms for $N=200, M=20, K=10$, and $\tau_{\mathrm{p}}=20$.

\subsection{MMSE-Based Channel Estimation}

The estimated effective channel matrix of the active UEs, $\hat{\mathbf{X}}_{\mathcal{S}}$, provided by the proposed approach can be used for coherent data detection. However, if the second-order channel statistics are known to the BS, a more accurate channel estimate can be obtained by applying the MMSE estimator.

Let us define the $\operatorname{vec}(\cdot)$ operation as the column-wise stacking of a matrix. We define $\mathbf{y}=\operatorname{vec}\left(\mathbf{Y}^{\mathrm{T}}\right) \in \mathbb{C}^{\tau_{\mathrm{p}} M}, \mathbf{w}=\operatorname{vec}\left(\mathbf{W}^{\mathrm{T}}\right) \in$ $\mathbb{C}^{\tau_{\mathrm{p}} M}$ and $\mathbf{x}=\operatorname{vec}\left(\mathbf{X}_{\mathcal{S}}\right) \in \mathbb{C}^{K M}$. Accordingly, 2) can be rewritten as!

$$
\mathbf{y}=\boldsymbol{\Theta} \mathbf{x}+\mathbf{w},
$$

where $\boldsymbol{\Theta}=\boldsymbol{\Phi}_{\mathcal{S}} \otimes \mathbf{I}_{M} \in \mathbb{C}^{M \tau_{\mathrm{p}} \times K M}$, and the operator $\otimes$ denotes the Kronecker product. The vectorization in 26 transforms the matrix estimation into a classical form of vector estimation which enables the use of the linear MMSE estimator given by [23. Eq. (12.26)]

$$
\mathbf{x}^{\mathrm{J}-\mathrm{MMSE}}=\operatorname{vec}\left(\mathbf{X}^{\mathrm{J}-\mathrm{MMSE}}\right)=\overline{\mathbf{x}}+\mathbf{R}_{\text {diag }} \boldsymbol{\Theta}^{\mathrm{H}} \mathbf{Q}(\mathbf{y}-\mathbf{\Theta} \overline{\mathbf{x}}),
$$

where $\mathbf{Q}=\left(\boldsymbol{\Theta} \mathbf{R}_{\text {diag }} \boldsymbol{\Theta}^{\mathrm{H}}+\sigma^{2} \mathbf{I}_{\tau_{\mathrm{p}} M}\right)^{-1}, \overline{\mathbf{x}}$ denotes the mean of $\mathbf{x}$, and $\mathbf{R}_{\text {diag }}$ denotes the covariance matrix of $\mathbf{x}$ given as a block diagonal matrix with the main-diagonal blocks are given by the scaled covariance matrices $\tilde{\mathbf{R}}_{i}$ corresponding to the active UEs $i \in \mathcal{S}$.

\section{NUMERICAL RESULTS}

Let us consider a single cell that consists of one BS equipped with $M$ antennas serving a total of $N=200$ uniformly distributed UEs, out of which only $K=10$ are active at each $T_{\text {c }}$. The channel between the $i$ th UE and the BS consists of $P_{i}=200$ paths with uniformly distributed angle of arrival $\psi_{i, p} \in\left[\frac{\pi}{3}, \frac{2 \pi}{3}\right]$. Each user $i \in \mathcal{N}$ is assigned a unique normalized quadratic phase-shift keying sequence $\phi_{i}$, with $\tau_{\mathrm{p}}=20$, generated from an i.i.d. complex Bernoulli distribution.

Channel estimation is quantified in terms of normalized mean square error (NMSE) defined as $\frac{\mathbb{E}\left[\left\|\mathbf{X}-\hat{\mathbf{X}}_{\mathcal{S}}\right\|_{\mathrm{F}}^{2}\right]}{\mathbb{E}\left[\|\mathbf{X}\|_{\mathrm{F}}^{2}\right]}$, where the expectation is computed via Monte-Carlo averaging over all sources of randomness. Thus, the NMSE is presented as the normalized average square error (NASE). User activity detection is quantified in terms of successful support recovery (SSR) rate defined as $\frac{|\mathcal{S} \cap \hat{\mathcal{S}}|}{|\mathcal{S}-\hat{\mathcal{S}}|+K}$, where $\hat{\mathcal{S}}$ denotes the detected support.

We compare the performance of cov-ADMM, IRW-ADMM, and ADMM, to two algorithms that solve the problem in (3), namely, fast alternating direction methods (F-ADM) [24] and SPARROW [3].

\footnotetext{
${ }^{1}$ We assume perfect UEs identification, as the main goal is to show channel estimation quality improvement gained by using the MMSE estimator.
}

In addition, we use genie-aided least square (LS) and genie-aided MMSE estimators that are provided "oracle" knowledge on the true set of active UEs to establish an optimal performance benchmark.

Fig. 1 a) presents the channel estimation performance in terms of NASE against SNR. First, in the case when the second-order statistics of the channels are not available at the BS, the proposed IRW-ADMM provides a significant improvement to the channel estimation quality compared to ADMM, ADM, and SPARROW. Furthermore, IRW-ADMM achieves a similar performance compared to oracle LS estimator. This result points out clearly the remarkable gain obtained by the iterative reweighted $\ell_{2,1}$-norm minimization approach. Second, if the BS is provided with the second-order statistics of the channels, the proposed cov-ADMM improves considerably the channel estimation. In fact, it provides the same performance as IRW-ADMM while using $10 \mathrm{~dB}$ lower SNR. Moreover, using the cov-ADMM with an MMSE estimator renders the same performance as the oracle MMSE estimator starting at SNR $=10 \mathrm{~dB}$.

Fig. 1 b) shows the user identification accuracy in terms of SSR rate against SNR. The results show that cov-ADMM indisputably provides the highest SSR rate amongst all the considered algorithms. In fact, cov-ADMM identifies the set of true active users perfectly for $\mathrm{SNR} \geq 10 \mathrm{~dB}$. In addition, the IRW-ADMM provides a significant improvement compared to ADMM, F-ADM, and SPARROW and it achieves an SSR rate $>0.95$ around $\mathrm{SNR}=8 \mathrm{~dB}$.

Fig. 11.c) shows the typical convergence behavior of the proposed algorithms at $\mathrm{SNR}=16 \mathrm{~dB}$. The results reveal that IRWADMM requires approximately 50 iterations to convergence. We note that the early iterations may find inaccurate signal estimates, hence, the lower performance when the number of iterations is less than 10. Furthermore, the proposed cov-ADMM convergence to its optimal solution in about 25 iterations. The results presented in Fig. 1 highlight clearly the significant gains obtained by exploiting available prior channel covariance information at the BS, as it yields the best performance in terms of channel estimation, user detection, and convergence rate.

\section{CONCLUSION}

The paper investigated joint support and signal recovery from an MMV model for the use case of user identification and channel estimation in MIMO-based grant-free mMTC. The paper proposed the formulation of JUICE based on an iterative reweighted $\ell_{2,1}$-norm minimization problem that exploits the second-order channel statistics when they are available to the BS. An ADMM-based algorithm was derived to provide a computationally efficient solution. The numerical results show significant improvement in UEs activity detection accuracy, channel estimation quality, and convergence rate. 


\section{REFERENCES}

[1] Ali Cagatay Cirik, Naveen Mysore Balasubramanya, Lutz Lampe, Gustav Vos, and Steve Bennett, "Toward the standardization of grant-free operation and the associated NOMA strategies in 3GPP," IEEE Commun. Stand. Mag., vol. 3, no. 4, pp. 60-66, 2019.

[2] Emmanuel J Candés, Justin Romberg, and Terence Tao, "Robust uncertainty principles: Exact signal reconstruction from highly incomplete frequency information," IEEE Trans. Inform. Theory, vol. 52, no. 2, pp. 489-509, Feb. 2006.

[3] Christian Steffens, Marius Pesavento, and Marc E Pfetsch, "A compact formulation for the $l_{2,1}$ mixed-norm minimization problem," IEEE Trans. Signal Processing, vol. 66, no. 6, pp. 1483-1497, 2018.

[4] Joel A Tropp, Anna C Gilbert, and Martin J Strauss, "Algorithms for simultaneous sparse approximation. part I: Greedy pursuit," Signal processing, vol. 86, no. 3, pp. 572-588, 2006.

[5] David L Donoho, Arian Maleki, and Andrea Montanari, "Message-passing algorithms for compressed sensing," Proc. Natl. Acad. Sci. U. S. A., vol. 106, no. 45, pp. 18914-18919, 2009.

[6] David P Wipf and Bhaskar D Rao, "An empirical Bayesian strategy for solving the simultaneous sparse approximation problem," IEEE Trans. Signal Processing, vol. 55, no. 7, pp. 3704-3716, 2007.

[7] Byeong Kook Jeong, Byonghyo Shim, and Kwang Bok Lee, "MAP-based active user and data detection for massive machine-type communications," IEEE Trans. Veh. Technol., vol. 67, no. 9, pp. 8481-8494, 2018.

[8] Yang Du, Cong Cheng, Binhong Dong, Zhi Chen, Xiaodong Wang, Jun Fang, and Shaoqian Li, "Block-sparsity-based multiuser detection for uplink grant-free NOMA," IEEE Trans. Wireless Commun., vol. 17, no. 12, pp. 7894-7909, 2018.

[9] Zhilin Chen, Foad Sohrabi, and Wei Yu, "Sparse activity detection for massive connectivity," IEEE Trans. Signal Processing, vol. 66, no. 7, pp. 1890-1904, 2018.

[10] Liang Liu and Wei Yu, "Massive connectivity with massive MIMO - part I: Device activity detection and channel estimation," IEEE Trans. Signal Processing, vol. 66, no. 11, pp. 2933-2946, 2018.

[11] Kamil Senel and Erik G Larsson, "Grant-free massive MTCenabled massive MIMO: A compressive sensing approach," IEEE Trans. Commun., vol. 66, no. 12, pp. 6164-6175, 2018.

[12] Malong Ke, Zhen Gao, Yongpeng Wu, Xiqi Gao, and Robert Schober, "Compressive sensing-based adaptive active user detection and channel estimation: Massive access meets massive MIMO,” IEEE Trans. Signal Processing, vol. 68, pp. 764-779, 2020.

[13] Xiaoxu Zhang, Ying-Chang Liang, and Jun Fang, "Novel Bayesian inference algorithms for multiuser detection in M2M communications," IEEE Trans. Veh. Technol., vol. 66, no. 9, pp. 7833-7848, 2017.

[14] Zhilin Chen, Foad Sohrabi, Ya-Feng Liu, and Wei Yu, "Covariance based joint activity and data detection for massive random access with massive MIMO," in Proc. IEEE Int. Conf. Commun., 2019, pp. 1-6.
[15] Emil Björnson, Luca Sanguinetti, and Merouane Debbah, "Massive MIMO with imperfect channel covariance information," in Proc. Annual Asilomar Conf. Signals, Syst., Comp., 2016, pp. 974-978.

[16] Emmanuel J Candés, Michael B Wakin, and Stephen P Boyd, "Enhancing sparsity by reweighted $\ell_{1}$ minimization," $J$. FOURIER Anal. Applic., vol. 14, no. 5-6, pp. 877-905, 2008.

[17] Li You, Xiqi Gao, Xiang-Gen Xia, Ni Ma, and Yan Peng, "Pilot reuse for massive MIMO transmission over spatially correlated Rayleigh fading channels," IEEE Trans. Wireless Commun., vol. 14, pp. 3352 -3366, 062015.

[18] Emil Björnson, Luca Sanguinetti, Henk Wymeersch, Jakob Hoydis, and Thomas L Marzetta, "Massive MIMO is a reality - what is next?: Five promising research directions for antenna arrays," Digit. Signal Process., vol. 94, pp. 3-20, 2019.

[19] Luca Sanguinetti, Emil Björnson, and Jakob Hoydis, “Towards massive MIMO 2.0: Understanding spatial correlation, interference suppression, and pilot contamination," IEEE Trans. Commun., vol. 68, no. 1, pp. 232-257, 2020.

[20] Stephen Boyd, Neal Parikh, Eric Chu, Borja Peleato, Jonathan Eckstein, et al., "Distributed optimization and statistical learning via the alternating direction method of multipliers," Foundations and Trends $\mathrm{R}$ in Machine learning, vol. 3, no. 1, pp. 1-122, 2011.

[21] Tom Goldstein, Christoph Studer, and Richard Baraniuk, "A field guide to forward-backward splitting with a FASTA implementation," arXiv preprint arXiv:1411.3406, 2014.

[22] Bharath K Sriperumbudur, David A Torres, and Gert RG Lanckriet, "A majorization-minimization approach to the sparse generalized eigenvalue problem," Mach. Lear., vol. 85, no. 1-2, pp. 3-39, 2011.

[23] Steven M Kay, Fundamentals of statistical signal processing, Prentice Hall PTR, 1993.

[24] Hongtao Lu, Xianzhong Long, and Jingyuan Lv, "A fast algorithm for recovery of jointly sparse vectors based on the alternating direction methods," in Proc. 14th Int. Conf. Artif. Intell. Statis., 2011, pp. 461-469. 